\title{
Parameter Optimization of Polishing M300 Mold Steel with an Elastic Abrasive
}

\author{
Xiao-Jun Wu $\mathbb{D}$, Xin Tong $\mathbb{D}$, Hao Sun, Huibo Jia, and Lu Zhang \\ School of Mechanical and Electrical Engineering, Xian University of Architecture and Technology, Xian, China \\ Correspondence should be addressed to Xiao-Jun Wu; wuxiaojun@xauat.edu.cn
}

Received 1 September 2018; Accepted 19 November 2018; Published 5 December 2018

Academic Editor: Liang Jing

Copyright (C) 2018 Xiao-Jun Wu et al. This is an open access article distributed under the Creative Commons Attribution License, which permits unrestricted use, distribution, and reproduction in any medium, provided the original work is properly cited.

In order to achieve high-quality polishing of M300 mold steel curved surface, an elastic abrasive is introduced in this paper, and its polishing parameters are optimized so that the mirror roughness can be achieved. Based on the Preston equation and Hertz contact theory, the theoretical material removal equation for surface polishing of elastic abrasives is obtained, and the polishing parameters to be optimized are as follows: particle size S, rotational speed Wt, cutting depth Ap, and feed speed Vf. The Taguchi method is applied to design the orthogonal experiment with four factors and three levels. The influence degree of various factors on the roughness of the polished surface and the combination of parameters to be optimized were obtained by the range analysis method. The particle swarm optimization algorithm optimizes the BP neural network algorithm (PSO-BP), which is used to optimize the polishing parameters. The results show that the rotational speed has the greatest influence on the roughness, the influence degree of abrasive particle size is greater than that of feed speed, and the influence of cutting depth is the least. The optimum parameters are as follows: particle size S 1200\#, rotational speed Wt 4500rpm, cutting depth Ap $0.25 \mathrm{~mm}$, and feed speed Vf $0.8 \mathrm{~mm} / \mathrm{min}$. The roughness of the surface polishing with optimum parameters is reduced to $0.021 \mu \mathrm{m}$.

\section{Introduction}

Due to its high Cr (16\%) content, M300 mold steel has a good corrosion resistance and a wear resistance and has strong resistance to the erosion of general chemicals such as industrial hydrochloric acid [1]. It is often used in the mold for various kinds of plastics, such as transparent plastics and camera lenses. As one of the most important processes of mold surface disposing, mold polishing directly decides the quality of the mold surface and determines its performance. At present, mold polishing mainly adopts traditional manual polishing, which is time-consuming and laborious, and the polishing quality is difficult to guarantee $[1,2]$.

In modern mold manufacturing, the proportions of free-form surfaces are increasing, and higher requirements of mold processing techniques are needed [3]. The elastic abrasive can have a good profiling contact with a curved surface workpiece on account of its polymer elastic abrasive binder structure with greater flexibility, which is different from the rigid fixed abrasive grinding wheel in which fretting of adjacent abrasives may happen on the partial surface [4].
So it is beneficial to improve the quality of curved surface polishing using an elastic abrasive.

The surface polishing mechanism and parameter optimization have been deeply studied. Jf. Zhang has studied the parameter optimization of five-axis polishing using an abrasive belt flap wheel for a blisk blade, in which RSM is used to analyze the interactions of polishing factors on SR and establish a predictive model between SR and various parameters [5]. A multiobjective particle swarm optimization algorithm (MOPSOA) is applied to optimize surface roughness of the workpiece after circular magnetic abrasive polishing by NhatTan Nguyen [6]. A statistics parameters optimization method based on index atlases is presented for a novel 5-DOF gasbag polishing machine tool by YB. Li [7]. However, for elastic abrasive polishing of M300 mold steel, there is still no complete study about parameter optimization.

In order to realize high-quality polishing of M300 mold steel curved surface, based on the Preston equation and Hertz contact theory, the polishing mechanism of the elastic abrasive is studied in this paper [8]. The automatic polishing experiment of M300 steel was carried out using elastic 
abrasive tools with various particle sizes. The influence of abrasive particle size, abrasive rotational speed, cutting depth, and feed speed on the surface roughness was analyzed.

The backpropagation (BP) neural network has strong adaptive and self-organizing capabilities and is widely used in data prediction and numerical analysis. The traditional BP neural network uses error backpropagation to adjust the connection weight of the network. The BP neural network can easily fall into the local optimal solution, and the convergence speed is slow and the network training is unstable. Therefore, the particle swarm optimization (PSO) algorithm is used to optimize the network weight and threshold to improve the network accuracy and convergence speed.

Then the BP neural network algorithm, which is optimized by the particle swarm optimization algorithm (PSO$\mathrm{BP}$ ), is used to achieve optimal parameter combination. Finally, the surface quality, which is polished under the conditions of optimal parameter combination, is verified by experiments.

\section{The Polishing Mechanism Using an Elastic Abrasive}

The mechanism in the polishing process by elastic abrasive tools is so complex that the elastic-plastic deformation of abrasive surface and the continuous wear of contact area lead to the decrease and fluctuation of the contact surface pressure [9]. The model of material removal for the polishing process can be established according to the Preston equation for the surface polishing by an axial feed abrasive on the selfrotating workpiece. The Preston equation is a commonly used empirical formula for material removal rate, which reveals that the material removal depth by a single abrasive grain is proportional to the relative pressure and line speed on the abrasive. The MRR of the grain in a unit length of track can be expressed by the following formula [10]:

$$
\frac{d h}{d l}=K_{p} \frac{V_{s}+V_{f}}{V_{f}} P
$$

$\mathrm{Kp}$ is the correction factor, which is related to the hardness of the workpiece and the abrasive grains as well as the abrasive grain size; $\mathrm{Vs}(\mathrm{m} / \mathrm{s})$ is the tangential line speed of the abrasive; $V_{\mathrm{f}}(\mathrm{mm} / \mathrm{min})$ is the axial feed speed along the workpiece; $\mathrm{P}(\mathrm{Pa})$ is the pressure on the contact zone.

According to the Hertz contact theory, the polishing process can be simplified as the contact situation between the rigid body (workpiece) and the elastic body (abrasive). The contact surface between the workpiece and abrasive tool is an ellipse as shown in Figure 1. The contact pressure submits to the elliptical Hertz distribution [11]:

$$
\mathrm{P}(\mathrm{y}, \mathrm{z})=-P_{0} \sqrt{1-\left(\frac{z}{a}\right)^{2}-\left(\frac{y}{b}\right)^{2}} .
$$

$P_{0}=3 F_{n} / 2 \pi a b$ is the center pressure in the contact zone; Fn $(\mathrm{N})$ is the contact force in polishing, as shown in Figure 2.

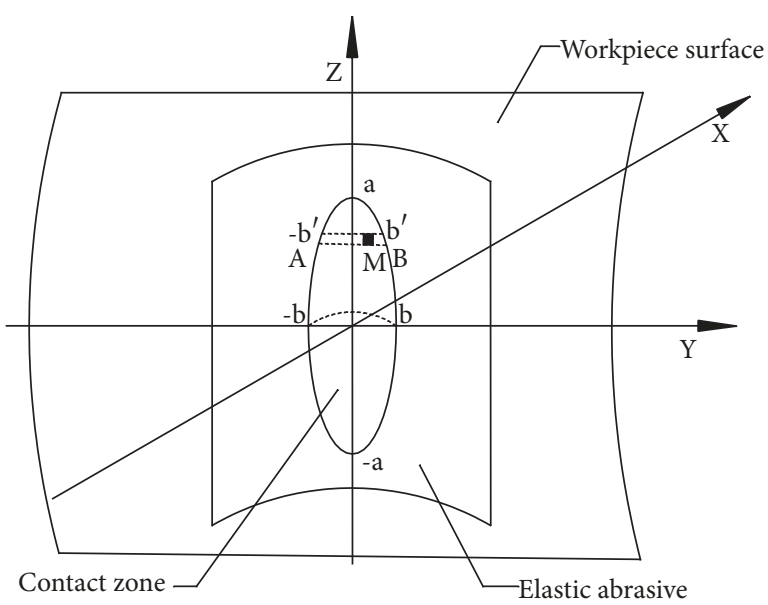

FIGURE 1: Contact institution of elastic abrasive and workpiece surface.

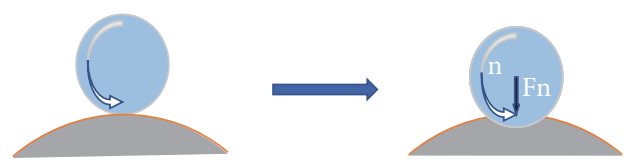

FIGURE 2: The contact force of elastic abrasive and workpiece surface.

The material removal amount of the infinitesimal $\mathrm{M}$ along the $\mathrm{Y}$ direction in contact region $\mathrm{AB}$ is

$$
\mathrm{h}(\mathrm{x})=\int_{-b^{\prime}}^{b^{\prime}} \frac{d h}{d l} d y=\int_{-b^{\prime}}^{b^{\prime}} K_{p} P \frac{V_{s} \pm V_{f}}{V_{f}} \mathrm{dy} .
$$

In the formula $\mathrm{b}^{\prime}=\mathrm{b} \sqrt{1-(x / c)^{2}}$.

The theoretical equation of MRR on the workpiece surface can be taken from formula (2) and formula (3):

$$
\mathrm{h}(\mathrm{x})=-K_{p} \frac{V_{s} \pm V_{f}}{V_{f}} \frac{3 F_{n}}{\pi a} \frac{x}{c} \sqrt{1-\left(\frac{x}{c}\right)^{2}} .
$$

Formula (4) shows that the MRR can be controlled by Vs, Vf, and Fn. The elastic abrasive can be attributed to the hyperelastic, and the contact pressure Fn of the workpiece surface is approximately proportional to the cutting depth of the abrasive tool [12]. During the surface polishing experiment, Vs reflects the grinding tool speed $\mathrm{Wt}$, Vf reflects the feed rate along the axis, and Ap stands for the setting cut depth of the abrasive tool. Therefore, the experiments are designed to optimize the polishing parameters $\mathrm{S}, \mathrm{Wt}, \mathrm{Ap}$, and Vf.

\section{Experiment}

3.1. Experimental Equipment. The polishing experiments were carried out on Mikoni 430P, a four-axis precision CNC machine. Just as shown in Figure 3(a), the device is composed of 3 moving axes $\mathrm{X}, \mathrm{Y}$, and $\mathrm{Z}$ and a rotation axis $\mathrm{A}$. The repeatability of positioning is $1 \mu \mathrm{m}$, and the maximum spindle 


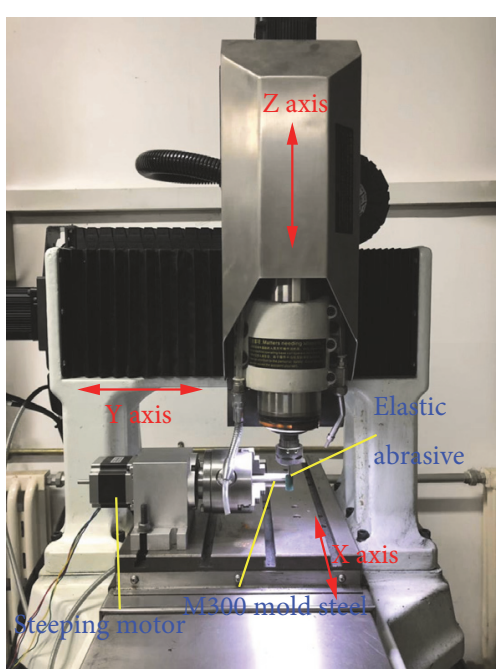

(a) Grinding and polishing process of elastic abrasive tool

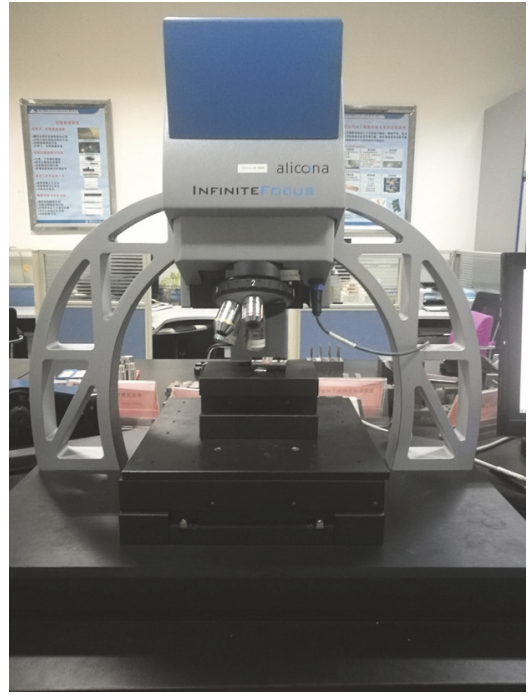

(b) Measurement of workpiece surface roughness

FIgURE 3: The experimental platform of polishing.

speed is $20000 \mathrm{rpm}$. The surface roughness $(\mathrm{Ra})$, which is the smaller pitches and unevenness of the tiny peaks and valleys of machined surface, was measured by an Alicona InfiniteFocus three-dimensional topography instrument, as shown in Figure 3(b).

3.2. Experimental Condition. The specimen was M300 mold steel $(\Phi 18 \times 55 \mathrm{~mm})$, and its chemical composition was shown in Table 1. The specimen is fixed on the worktable A-axis, and the rotating speed of the worktable is constant to $300 \mathrm{r} / \mathrm{min}$. The specific polishing conditions were shown in Table 2.

\subsection{Experimental Design and Results. Taking into account} the interaction among the factors, an orthogonal experiment with four factors and three levels [10] was designed based on the Taguchi method [13], which is shown in Table 3. The processing time of each group of experiments is 180s. In order to reduce the processing error, each group of experiments is processed three times. The result is taken as its average value, which is as shown in Table 4.

In Table 4, mean 1 is the mean of the normal variance of the surface roughness of the influencing factor in the level 1 combination.

Mean 2 is the mean of the normal variance of the surface roughness of the influencing factor in the level 2 combination.

Mean 3 is the mean of the normal variance of the surface roughness of the influencing factor in the level 3 combination.

The combination of grinding parameters for a single optimized target can be achieved by the signal-to-noise ratio (SNR) analysis of the experimental data. Since the optimization target is the surface roughness (Ra), the design parameters of the small characters are adopted, such as

$$
\mathrm{SNR}=-10 \lg \sum_{i=1}^{n} R_{i}^{2}
$$

TABLE 1: The chemical composition of the M300 steel.

\begin{tabular}{lccccc}
\hline Name & C & Cr & Mo & Mn & Si \\
\hline Content & 0.38 & 16.00 & 1.00 & 0.4 & 0.40 \\
\hline
\end{tabular}

Table 5 is the average response of SNR to $\mathrm{Ra}$ in each parameter level. The larger the SNR is, the higher the parameter influence on Ra will be. It can be seen that the abrasive grain size and the abrasive speed have a high influence on Ra.

In order to express the influence trend of each factor level on the surface roughness more intuitively, the main effect diagram of polished roughness is obtained.

When the particle size is too small, the residual peak on the surface of the workpiece is not sufficiently cut, so that when the particle size is increased, the roughness is decreased. When the grinding speed is too fast, this results in incomplete cutting. However, too slow speed will result in a decrease in the number of abrasive grains involved in cutting per unit time. When the depth of cut increases, the roughness decreases due to overcutting of the abrasive grains. As the depth of cut further increases, the deformation of the abrasive increases and the contact area with the workpiece increases. Finally, the time of cutting of the abrasive is increased, and the roughness decreases. Excessive feed rates and low feed rates will result in undercutting and overcutting, respectively.

As shown in Figure 4, particle size S, grinding speed Wt, cutting depth AP, and feed rates Vf are the minimum roughness at levels $3,1,3$, and 2, respectively. Because the roughness is a small feature, the minRa parameter combinations A3 B1 C3 D2 of all levels can be obtained as the parameter combinations to be optimized. 
TABLE 2: Experimental conditions for grinding and polishing.

\begin{tabular}{lc}
\hline Name & Conditions \\
\hline Specification of abrasive tools & $\Phi 10$ mm silicone rubber based elastic abrasives \\
\hline Abrasive and particle size & Silicon carbide (carborundum), 320\#, 600\#, 1000\# \\
\hline Cooling-down methods & Dry polishing \\
\hline
\end{tabular}

TABLE 3: Factors and levels of the orthogonal experiment.

\begin{tabular}{lcccc}
\hline Processing parameters & \multicolumn{2}{c}{ Level } & 2 & 3 \\
\hline Particle size S (\#) & 1 & 600 & \\
\hline Abrasive tool speed Wt (r/min) & 320 & 6000 & \\
\hline Setting cut depth Ap (mm) & 4500 & 0.2 & 7500 \\
\hline Feed rate Vf & 0.1 & 1 & \\
\hline
\end{tabular}

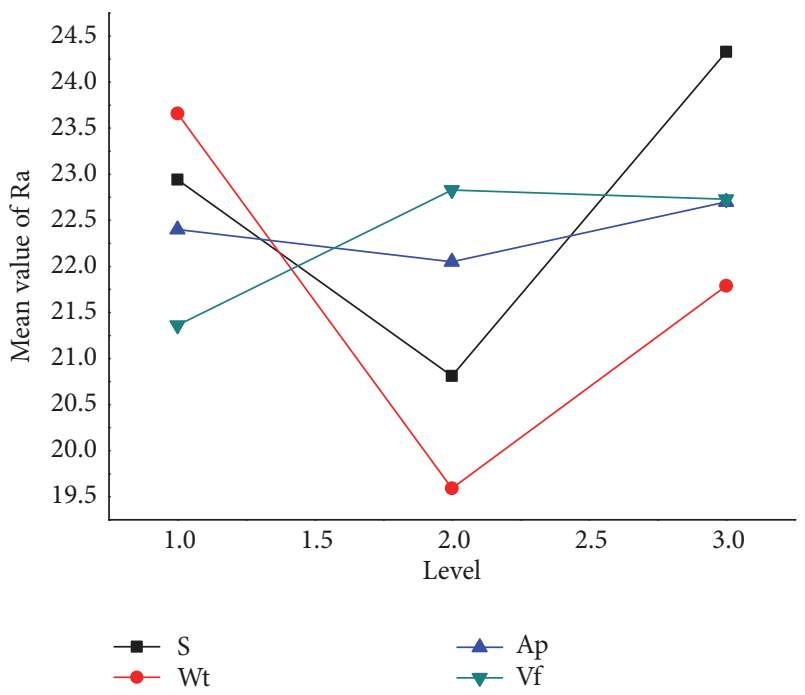

FIGURE 4: Main effect diagram of Ra.

\section{Parameter Optimization}

4.1. PSO-BP Neural Network Model. The surface roughness polished with an elastic abrasive is affected by many factors, and the complex nonlinear relationship between roughness and influencing factors is difficult to be fitted by a linear model or common nonlinear model. The BP neural network has high mapping ability and can realize any nonlinear mapping from input to output. By using the high mapping ability and generalization ability of the BP neural network, the mapping model between particle size $S$, rotational speed $\mathrm{Wt}$, cutting depth Ap, feed speed Vf, and polished surface roughness can be established to solve the problem of parameter optimization. However, the BP neural network can easily fall into the local extremum [14].

Particle swarm optimization (PSO) is a swarm intelligence optimization algorithm, which finds out the optimal region in a complex search space by the interaction among particles [15]. The learning of the BP neural network is mainly reflected in the adjustment process of the weight value and the threshold. The optimization operation of particle

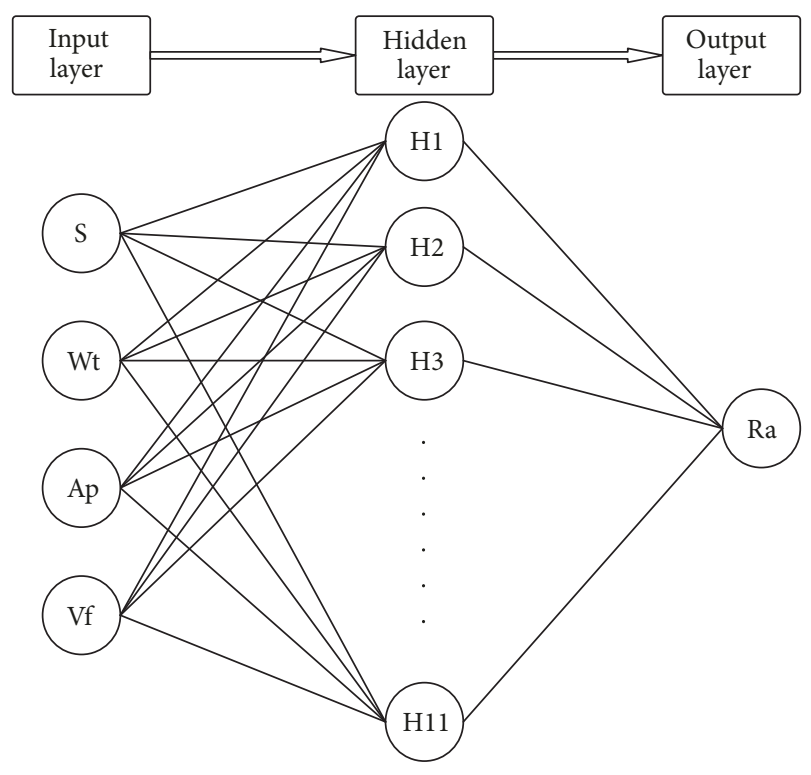

FIGURE 5: Schematic diagram of BP neural network structure.

swarm optimization corresponds to the weight value and the threshold of BP neural network algorithm, and then the PSOBP neural network model is established.

The particle size $\mathrm{S}$, rotational speed $\mathrm{Wt}$, cutting deep Ap, and feed speed Vf are input factors. The polishing surface roughness is used as the output factor. The BP neural network model with 1 hidden layer is established, as shown in Figure 5. The number of neurons in the hidden layer is 11. The transfer function of the hidden layer is tansig. The transfer function of the output layer is pureline. The training function is trainlm. The training accuracy, learning rate, and cycle times are 0.0001, 0.05, and 3000, respectively.

When the weight is optimized by particle swarm optimization, the connection weights of each layer of the neural network are encoded into a particle and the fitness is the mean square error of network output. Search for the optimal network weights within the default number of iterations. 
TABLE 4: Results of the orthogonal experiment.

\begin{tabular}{|c|c|c|c|c|c|c|}
\hline No. & Particle size S (A) & Grinding speed Wt (B) & Setting cut depth Ap (C) & Feed rate Vf (D) & Surface roughness $(\mathrm{Ra})$ & SNR \\
\hline 1. & 320 & 4500 & 0.1 & 0.5 & 0.037 & 28.64 \\
\hline 2. & 320 & 4500 & 0.2 & 1 & 0.074 & 22.62 \\
\hline 3. & 320 & 4500 & 0.3 & 2 & 0.088 & 21.11 \\
\hline 4. & 320 & 6000 & 0.3 & 0.5 & 0.069 & 23.22 \\
\hline 5. & 320 & 7500 & 0.3 & 2 & 0.030 & 30.46 \\
\hline 6. & 600 & 4500 & 0.1 & 1 & 0.079 & 22.05 \\
\hline 7. & 600 & 4500 & 0.2 & 2 & 0.059 & 24.58 \\
\hline 8. & 600 & 7500 & 0.3 & 1 & 0.072 & 22.85 \\
\hline 9. & 600 & 4500 & 0.2 & 2 & 0.055 & 25.19 \\
\hline 10. & 600 & 4500 & 0.3 & 0.5 & 0.108 & 19.33 \\
\hline 11. & 600 & 6000 & 0.1 & 2 & 0.117 & 18.64 \\
\hline 12. & 600 & 6000 & 0.2 & 0.5 & 0.145 & 16.77 \\
\hline 13. & 600 & 7500 & 0.2 & 1 & 0.139 & 17.14 \\
\hline 14. & 600 & 7500 & 0.3 & 2 & 0.106 & 19.49 \\
\hline 15. & 1000 & 4500 & 0.2 & 0.5 & 0.037 & 28.64 \\
\hline 16. & 1000 & 4500 & 0.3 & 2 & 0.047 & 26.56 \\
\hline 17. & 1000 & 4500 & 0.1 & 0.5 & 0.072 & 22.85 \\
\hline 18. & 1000 & 4500 & 0.2 & 1 & 0.046 & 26.74 \\
\hline 19. & 1000 & 7500 & 0.2 & 1 & 0.035 & 29.12 \\
\hline 20. & 1000 & 7500 & 0.1 & 1 & 0.045 & 26.94 \\
\hline 21. & 1000 & 7500 & 0.2 & 2 & 0.111 & 19.09 \\
\hline 22. & 1000 & 7500 & 0.3 & 0.5 & 0.105 & 19.58 \\
\hline 23. & 320 & 7500 & 0.2 & 0.5 & 0.294 & 10.63 \\
\hline 24. & 600 & 4500 & 0.1 & 1 & 0.083 & 21.62 \\
\hline 25. & 600 & 6000 & 0.3 & 1 & 0.094 & 20.54 \\
\hline 26. & 600 & 7500 & 0.1 & 0.5 & 0.074 & 22.62 \\
\hline 27. & 1000 & 4500 & 0.1 & 2 & 0.107 & 19.41 \\
\hline 28. & 600 & 6000 & 0.1 & 1 & 0.115 & 18.79 \\
\hline 29. & 600 & 4500 & 0.3 & 1 & 0.082 & 21.72 \\
\hline 30. & 320 & 4500 & 0.3 & 1 & 0.064 & 23.88 \\
\hline
\end{tabular}

TABLE 5: Signal-to-noise ratio (SNR) to surface roughness.

\begin{tabular}{cccccc}
\hline \multicolumn{2}{c}{ Parameter } & $\mathrm{S}$ & $\mathrm{Wt}$ & $\mathrm{Ap}$ & $\mathrm{Vf}$ \\
\hline \multirow{3}{*}{ Level } & 1 & 22.94 & $\underline{23.66}$ & 22.40 & 21.36 \\
& 2 & 20.81 & 19.59 & 22.05 & $\underline{22.83}$ \\
& 3 & $\underline{24.33}$ & 21.79 & $\underline{22.70}$ & 22.73 \\
\hline
\end{tabular}

The PSO algorithm is to find the optimal solution in a group of particles by iterating. The particle is updated by the $P_{\text {best }}$ values and the $G_{\text {best }}$ values. $P_{\text {best }}$ is the best location which is searched by particles. $G_{\text {best }}$ is the best location which is searched by the whole particle swarm.

Supposing $\mathrm{z}_{\mathrm{i}}=\left(\mathrm{z}_{\mathrm{i} 1}, \mathrm{z}_{\mathrm{i} 2}, \ldots, \mathrm{z}_{\mathrm{id}}, \ldots \mathrm{z}_{\mathrm{iD}}\right)$ is the D-dimensional position vector of the No. i particle, the position of the particle can be measured by the fitness function. $v_{i}=\left(v_{i 1}, v_{i 2}, \ldots, v_{i d}, \ldots, v_{i D}\right)$ is the fly velocity of particle $i$. $\mathrm{p}_{\mathrm{i}}=\left(\mathrm{p}_{\mathrm{i} 1}, \mathrm{p}_{\mathrm{i} 2}, \ldots, \mathrm{p}_{\mathrm{id}}, \ldots, \mathrm{p}_{\mathrm{iD}}\right)$ is the optimal position of the particle $\mathrm{i}$ so far. $\mathrm{p}_{\mathrm{g}}=\left(\mathrm{p}_{\mathrm{g} 1}, \mathrm{p}_{\mathrm{g} 2}, \ldots, \mathrm{p}_{\mathrm{gd}}, \ldots, \mathrm{p}_{\mathrm{gD}}\right)$ is the optimal position found so far by the particle swarm. The fly velocity and position are updated according to

$$
\begin{aligned}
v_{i d}^{k+1}=w v_{i d}^{k}+c_{1} r_{1}\left(p_{i d}-z_{i d}^{k}\right)+c_{2} r_{2}\left(p_{g d}-z_{i d}^{k}\right) \\
i=1,2, \ldots, m, d=1,2, \ldots, D .
\end{aligned}
$$

$\mathrm{K}$ : current number of iterations

$\mathrm{r}_{1}, \mathrm{r}_{2}:$ random number $[0,1]$

$\mathrm{c}_{1}, \mathrm{c}_{2}$ : acceleration constant

$\mathrm{W}$ : inertia weight

In order to maintain the equilibrium of particle swarm convergence speed and convergence efficiency, the initial algorithm should have a large global search capability, and the latter algorithm should have strong local search capability. Therefore, the linear variation of (7), (8), and (9) is used to improve the global optimization ability of the particle group 


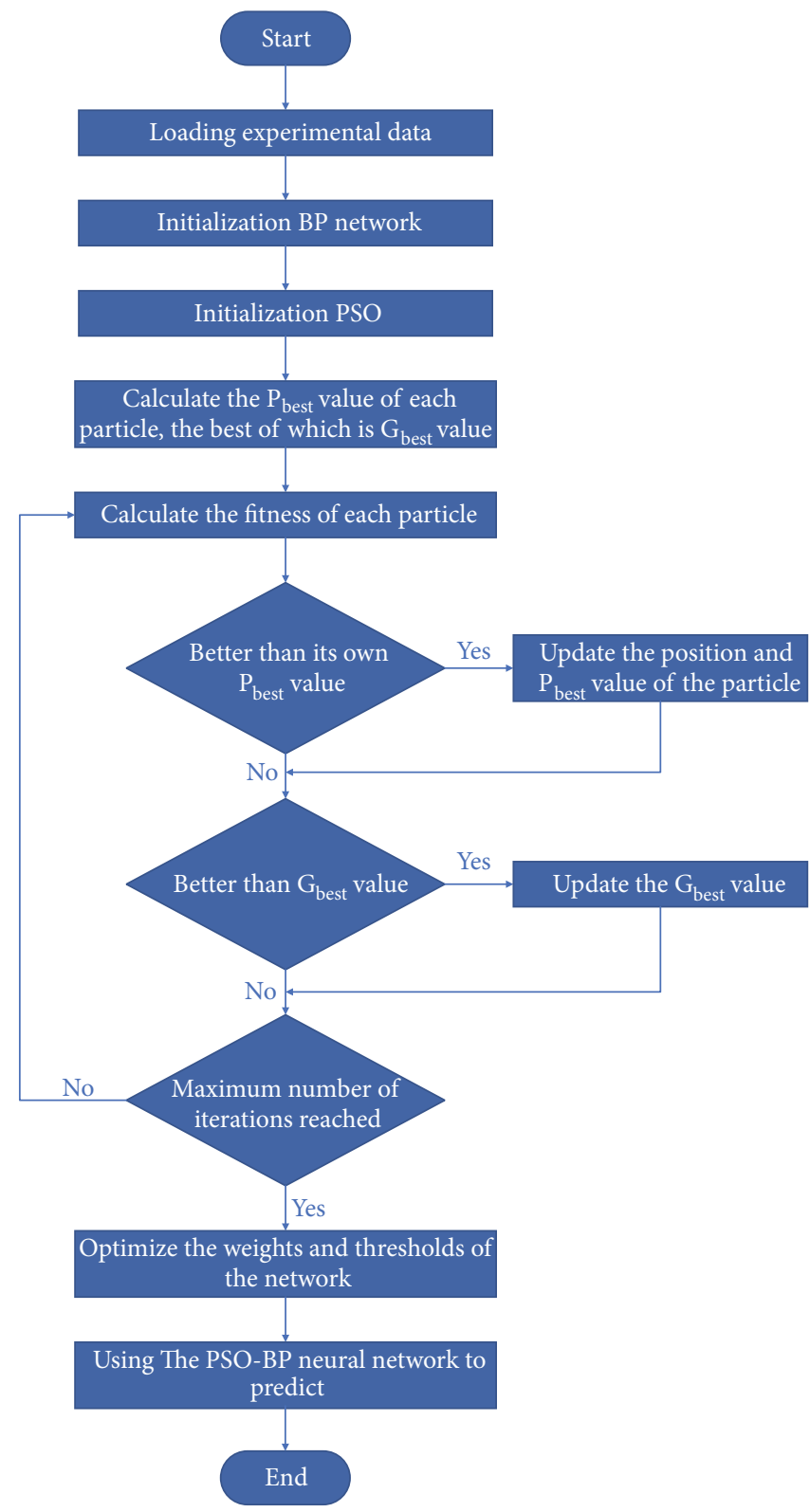

FIGURE 6: Flow-process diagram of PSO-BP.

at the initial stage and improve the local optimization ability of the particle group in the later stage.

$$
\begin{aligned}
& c_{1}=\left(c_{1 f}-c_{1 i}\right) \times\left(k \div k_{\max }\right)+c_{1 i} \\
& c_{2}=\left(c_{2 f}-c_{2 \mathrm{i}}\right) \times\left(k \div k_{\max }\right)+c_{2 i} \\
& \mathrm{w}=\mathrm{w}_{\max }-\frac{\mathrm{w}_{\max }-\mathrm{w}_{\min }}{\mathrm{k}_{\max }} \times \mathrm{k} .
\end{aligned}
$$

In general, when $C_{1}+C_{2}<4$, the optimization ability of the example group is the best [15], so $c_{1 \mathrm{f}}$ and $\mathrm{c}_{1 \mathrm{i}}$ are 0.5 and 2.5 , respectively; $c_{2 \mathrm{f}}$ and $\mathrm{c}_{2 \mathrm{i}}$ are 2.5 and 0.5 , respectively. $\mathrm{w}_{\max }$ and $\mathrm{w}_{\mathrm{min}}$ are 0.9 and 0.4 , respectively.

Set the maximum speed as 0.8 , the number of particles as 40 , and the minimum error as 0.001 . Build a PSO-BP network model (Figure 6) to train the data for rows 1-25 in Table 4 . The data of 26-30 rows is used to examine the trained network model. The comparison between the PSO-BP neural network and the BP neural network is shown in Figure 7.

Compared with Figure (a) and Figure (b), it can be seen that the PSO-BP neural network converges to the preset precision in only 6 steps, and the efficiency of the PSO-BP neural network is obviously improved compared with the basic BP neural network. By comparing Figure (c) with Figure (d), the predicted value of the former is very close to the experimental value, but the latter has a large deviation.

Just as shown in Table 6, the prediction error of the PSOBP network model is within $0.3 \%$. So the PSO-BP network model has a high accuracy, which can be used as a prediction model. 


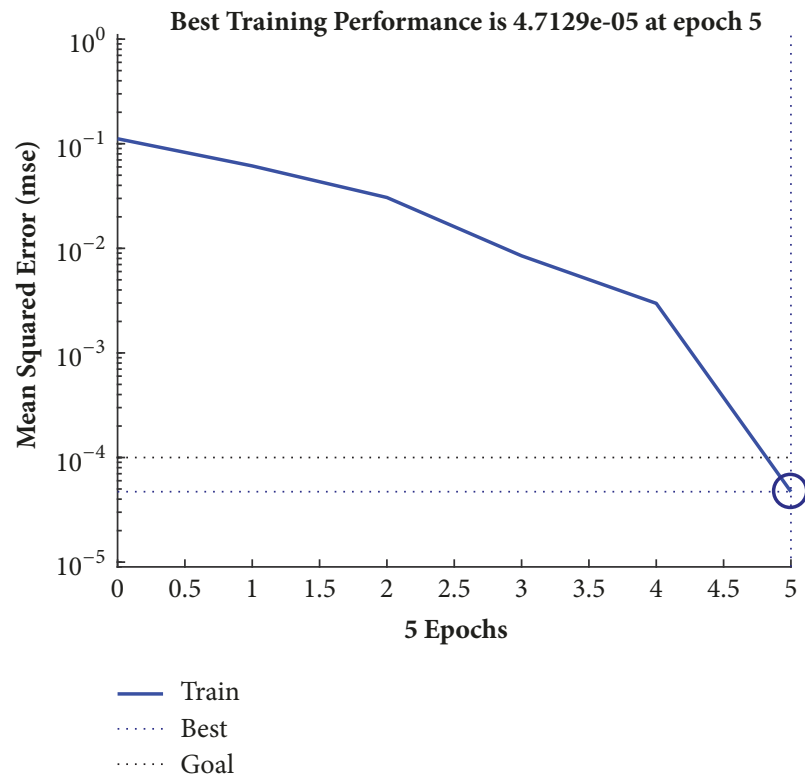

(a) The training process of PSO-BP neural network algorithm

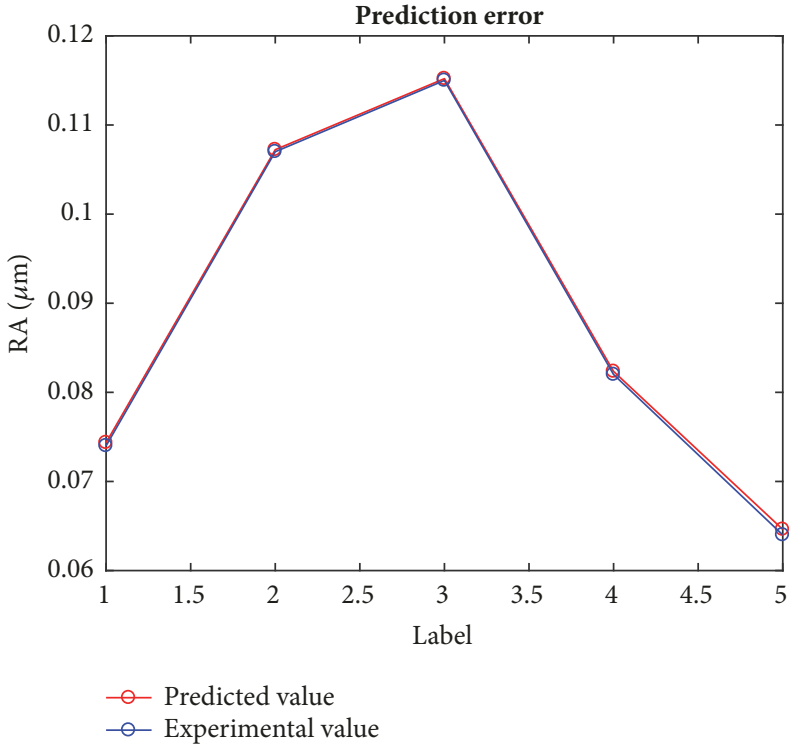

(c) Prediction error of PSO-BP neural network algorithm

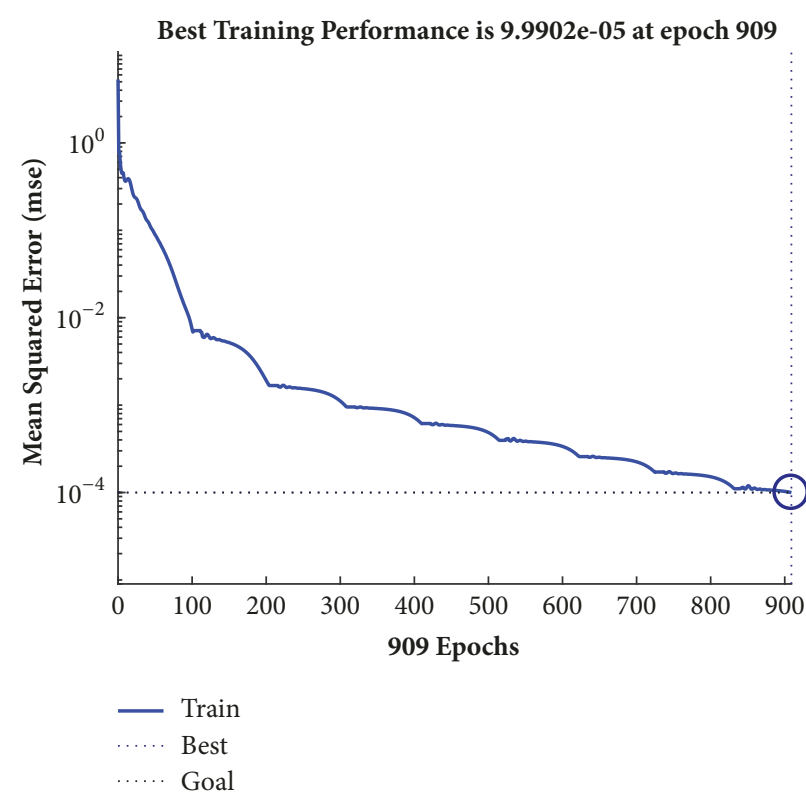

(b) The training process of basic BP neural network

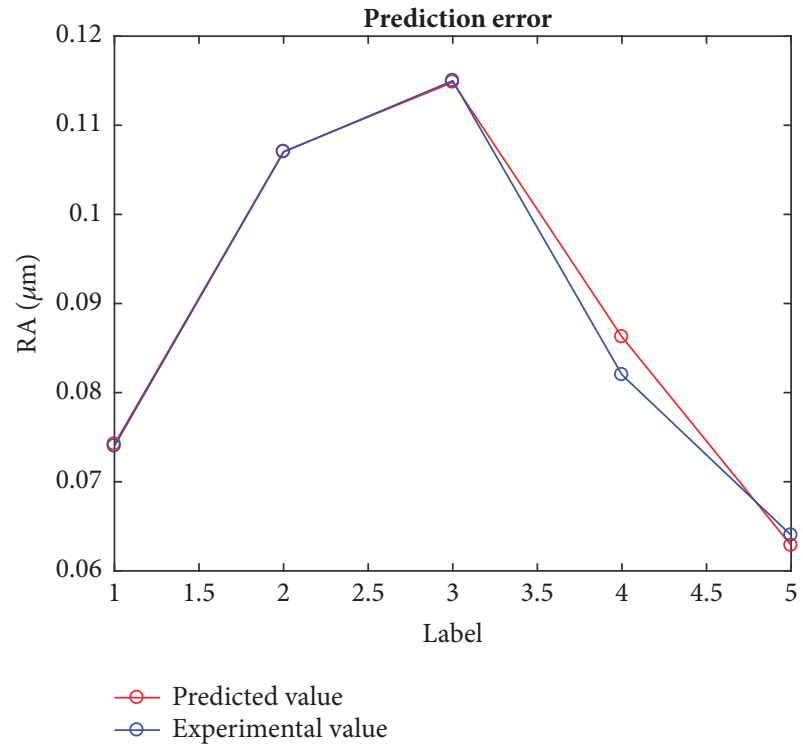

(d) Prediction error of basic BP neural network algorithm

FIgURE 7: The comparison of PSO-BP and BP.

TABLE 6: Predicted error.

\begin{tabular}{lccccc}
\hline No. & 26 & 27 & 28 & 29 & 30 \\
\hline Actual value $(\mu \mathrm{m})$ & 0.074 & 0.107 & 0.115 & 0.082 & 0.064 \\
\hline Predicted value $(\mu \mathrm{m})$ & 0.0741 & 0.1067 & 0.1148 & 0.0818 & 0.0643 \\
\hline Error $(\%)$ & 0.14 & 0.28 & 0.17 & 0.24 & 0.47 \\
\hline
\end{tabular}

4.2. Optimization Results. In this paper, a large number of experimental data are used to train the neural network, and a prediction model is established. Therefore, only the parameters set in the project can be brought into the model to obtain the optimal combination of polishing parameters.
In order to illustrate the feasibility of the algorithm in engineering applications, based on the minRa parameter combination of each factor, each factor is set to be 5 levels, and the distribution is shown in Table 7. The orthogonal test is designed by using the Taguchi method and the data is input into the trained PSO-BP neural network model for prediction. The results are shown in Table 8.

4.3. Experimental Verification. In Table 8, the optimized polishing parameter combination is obtained as follows: A5 B3 C2 D1 (S: 1200\#, Wt: 4500rpm, Ap: $0.25 \mathrm{~mm}$, and Vf: $0.8 \mathrm{~mm} / \mathrm{min}$ ). The confirmatory experiments of minRa 
TABLE 7: The distribution of each factor.

\begin{tabular}{lccccc}
\hline \multirow{2}{*}{ Processing parameters } & \multicolumn{5}{c}{ Level } \\
& 1 & 2 & 3 & 4 & 5 \\
\hline Particle size S (\#) & 700 & 800 & 1000 & 1100 & 1200 \\
\hline Abrasive tool speed Wt (r/min) & 4300 & 4400 & 4500 & 4600 & 4700 \\
\hline Setting cut depth Ap (mm) & 0.2 & 0.25 & 0.3 & 0.35 & 0.4 \\
\hline Feed rateVf (mm/min) & 0.8 & 0.9 & 1 & 1.1 & 1.2 \\
\hline
\end{tabular}

TABLE 8: Predicted results.

\begin{tabular}{lccccc}
\hline No. & $\mathrm{A}$ & $\mathrm{B}$ & $\mathrm{C}$ & $\mathrm{D}$ & $\mathrm{Ra}$ \\
\hline 1. & 700 & 4300 & 0.2 & 0.8 & 0.0850 \\
\hline 2. & 700 & 4400 & 0.25 & 0.9 & 0.0971 \\
\hline 3. & 700 & 4500 & 0.3 & 1 & 0.0761 \\
\hline 4. & 700 & 4600 & 0.35 & 1.1 & 0.0637 \\
\hline 5. & 700 & 4700 & 0.4 & 1.2 & 0.0891 \\
\hline 6. & 800 & 4300 & 0.25 & 1 & 0.0896 \\
\hline 7. & 800 & 4400 & 0.3 & 1.1 & 0.0702 \\
\hline 8. & 800 & 4500 & 0.35 & 1.2 & 0.0705 \\
\hline 9. & 800 & 4600 & 0.4 & 0.8 & 0.0621 \\
\hline 10. & 800 & 4700 & 0.2 & 0.9 & 0.0611 \\
\hline 11. & 1000 & 4300 & 0.3 & 1.2 & 0.0491 \\
\hline 12. & 1000 & 4400 & 0.35 & 0.8 & 0.0600 \\
\hline 13. & 1000 & 4500 & 0.4 & 0.9 & 0.0729 \\
\hline 14. & 1000 & 4600 & 0.2 & 1 & 0.0456 \\
\hline 15. & 1000 & 4700 & 0.25 & 1.1 & 0.0438 \\
\hline 16. & 1100 & 4300 & 0.35 & 0.9 & 0.0548 \\
\hline 17. & 1100 & 4400 & 0.4 & 1 & 0.0634 \\
\hline 18. & 1100 & 4500 & 0.2 & 1.1 & 0.0393 \\
\hline 19. & 1100 & 4600 & 0.25 & 1.2 & 0.0360 \\
\hline 20. & 1100 & 4700 & 0.3 & 0.8 & 0.0414 \\
\hline 21. & 1200 & 4300 & 0.4 & 1.1 & 0.0460 \\
\hline 22. & 1200 & 4400 & 0.2 & 1.2 & 0.0339 \\
\hline 23. & 1200 & 4500 & 0.25 & 0.8 & 0.0211 \\
\hline 24. & 1200 & 4600 & 0.3 & 0.9 & 0.0392 \\
\hline 25. & 1200 & 4700 & 0.35 & 1 & 0.0536 \\
\hline & & & & & \\
\hline . & 1000 & & & \\
\hline
\end{tabular}

parameter combination A3 B1 C3 D2 and optimized parameter combination A5 B3 C2 D1 are carried out, respectively.

The comparison of the surface morphologies of the M300 workpiece before and after polishing under the condition of optimized parameter combination A5 B3 C2 D1 is shown in Figure 9(b). It can be seen that the polishing pattern is obviously reduced and the surface damage is greatly improved. The surface roughness $\mathrm{Ra}$ is reduced to $0.021 \mu$ $\mathrm{m}$ after machining. Compared with the minRa parameter combination (as shown in Figure 8), the roughness is reduced significantly, and the surface quality is improved obviously, which mean that the parameter optimization method used is feasible.

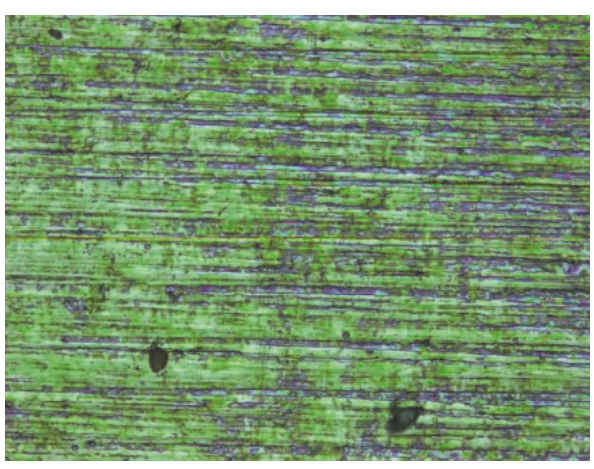

FIGURE 8: Surface topographies of the workpiece by minRa parameter combination $(\mathrm{S}=1000 \#, \mathrm{Wt}=4500 \mathrm{r} / \mathrm{min}, \mathrm{Ap}=0.3 \mathrm{~mm}$, and $\mathrm{Vf}=1 \mathrm{~mm} / \mathrm{min}$ ).

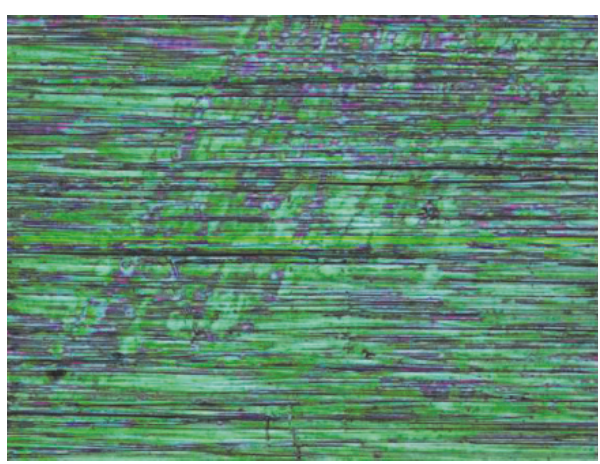

(a) Initial surface morphology

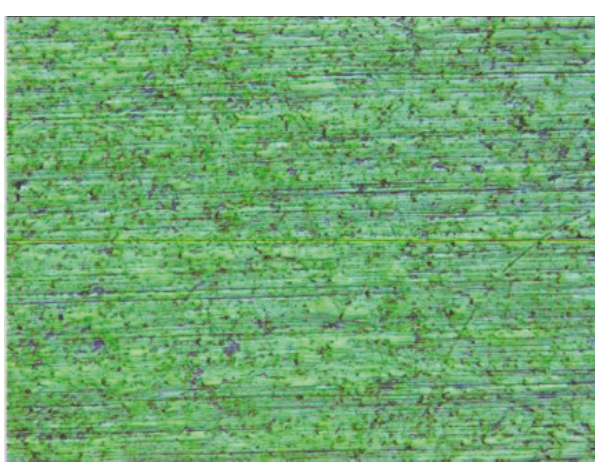

(b) Surface morphology polished

FIgURE 9: Surface topographies of the workpiece by optimized parameters before and after polishing $(S=1200 \#, W t=4500 \mathrm{r} / \mathrm{min}$, $\mathrm{Ap}=0.25 \mathrm{~mm}$, and $\mathrm{Vf}=0.8 \mathrm{~mm} / \mathrm{min}$ ).

\section{Conclusion}

Compared with the polishing of free and consolidated abrasive hard grinding wheels, a silicon carbide abrasive as well as a silicone rubber based elastic abrasive is cheaper and has a better profile when polishing the curved surface of the M300 mold steel. It can easily obtain high surface quality and provides a feasible method for high efficiency and highquality polishing of M300 mold steel.

(1) Based on the parameter combination of particle size, grinding speed, cutting depth, and feed speed, the orthogonal 
experiment is carried out, and the range analysis of experimental results is acted. The result shows that the speed of grinding tool has the greatest influence on roughness, and the influence of particle size and feed speed on roughness is close. The influence degree of cutting depth is the least. The minRa parameters of each level are as follows: S 1000\#, Wt 4500rpm, Ap $0.3 \mathrm{~mm}$, and Vf $1 \mathrm{~mm} / \mathrm{min}$

(2) The experimental parameters are trained and examined by the PSO-BP neural network algorithm. The results show that the prediction roughness error is less than $0.3 \%$, which means that the network structure has high precision

(3) The surface roughness is taken as the optimization indexes. Based on the combination of minRa parameters, the polishing parameters are optimized by using the trained PSO-BP neural network structure. The optimization results show that the optimal parameter combination is S 1200\#, Wt $4500 \mathrm{rpm}$, Ap $0.25 \mathrm{~mm}$, and Vf $0.8 \mathrm{~mm} / \mathrm{min}$. The verified experiment shows that the roughness of the polished surface is reduced to $0.021 \mu \mathrm{m}$ under the optimal parameter combination condition, which is consistent with the predicted optimization results. The parameter optimization method based on the PSO-BP neural network algorithm is feasible to optimize the polishing parameters

\section{Data Availability}

The data used to support the findings of this study are available from the corresponding author upon request.

\section{Conflicts of Interest}

The authors declare that they have no conflicts of interest.

\section{Acknowledgments}

This project is financially supported by the National Natural Science Foundation of China (Grant No. 51375361 and No. 51475353). The authors are also grateful for the Industrial Research Projects of Shaanxi Province (2012K09-15).

\section{References}

[1] T. Zhang, P. Li, Z. Liu, and N. Liu, "Failure Analysis of M300 Steel for Zn Alloy Die Casting Nozzles," Special Casting \& Nonferrous Alloys, vol. 26, no. 4, pp. 226-228, 2006.

[2] P.-Y. L. Bail, A. Depince, N. Chenais, S. Mahe, G. Maisse, and C. Labbe, "Optimization of pre-polishing parameters on a 5-axis milling machine," BMC Developmental Biology, vol. 10, article no. $64,2010$.

[3] M. J. Tsai and J. F. Huang, "Efficient automatic polishing process with a new compliant abrasive tool," The International Journal of Advanced Manufacturing Technology, vol. 30, no. 9, pp. 817-827, 2006.

[4] W. Wei, Research on Progressive Soft-bonded Abrasive Grain Pneumatic Wheel Finishing, Zhejiang University of Technology, Hangzhou, China, 2016.

[5] J. Zhang, Y. Shi, X. Lin, and Z. Li, "Parameter optimization of five-axis polishing using abrasive belt flap wheel for blisk blade,"
Journal of Mechanical Science and Technology, vol. 31, no. 10, pp. 4805-4812, 2017.

[6] N. Nguyen, S. Yin, F. Chen, H. Yin, V. Pham, and T. Tran, "Multiobjective optimization of circular magnetic abrasive polishing of SUS304 and Cu materials," Journal of Mechanical Science and Technology, vol. 30, no. 6, pp. 2643-2650, 2016.

[7] Y. Li, D. Tan, D. Wen, S. Ji, and D. Cai, "Parameters optimization of a novel 5-DOF gasbag polishing machine tool," Chinese Journal of Mechanical Engineering, vol. 26, no. 4, pp. 680-688, 2013.

[8] S. M. Ji, W. Li, D. P. Tan et al., "Processing Characteristics of Soft Abrasive Flow Based on Preston Equation," Chinese Journal of Mechanical Engineering, vol. 47, no. 17, pp. 156-163, 2011.

[9] Y. Huang, C. Yang, and Z. Huang, "Experimental research on abrasive belt grinding for 304 stainless steel," China Mechanical Engineering, vol. 22, no. 3, pp. 291-295, 2011.

[10] L. Zhang and C. M. Yuan, "Modeling and Experimental Research on Surface Removal of Die Surface Polishing," Chinese Journal of Mechanical Engineering, vol. 38, no. 12, pp. 98-102, 2002.

[11] L. Valentine, The Principles of Contact Mechanics and Tribology and Their Applications, Tsinghua University Press, Beijing, China, 2011.

[12] Z. K. Xu, Stainless Steel Thin-walled Workpieces Automatic Mechanical Polishing Technology Research and Process Optimization, Huazhong University of Science and Technology Press, Wuhan, China, 2012.

[13] X. B. Xu and H. Shao, "Multi-objective Optimization of Turning ParametersBased on Taguchi Algorithm and Grey Relational Theory," Tool Technology, vol. 49, no. 8, pp. 15-18, 2015.

[14] M. Li, Research on Tool Wear Monitoring Technology Based on Particle Swarm Optimization Neural Network, Southwest Jiaotong University, Chengdu, China, 2012.

[15] R. C. Eberhart and J. Kennedy, "A new optimizer using particle swarm theory," in Proceedings of the 6th International Symposium on Micromachine and Human Science, pp. 39-43, IEEE, Nagoya, Japan, October 1995. 


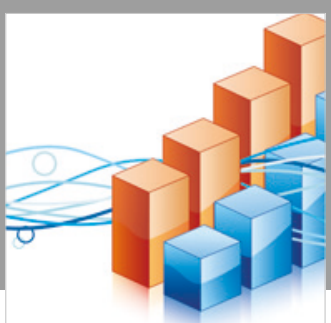

Advances in

Operations Research

\section{-n-m}
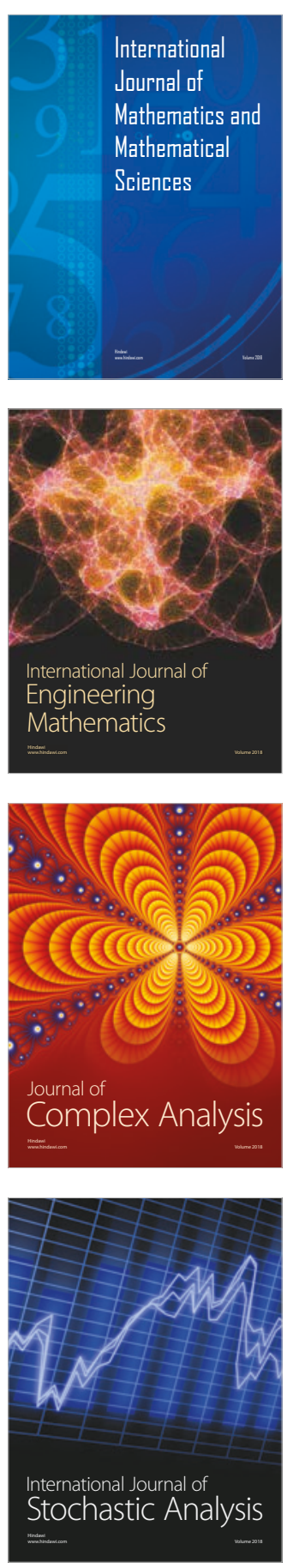
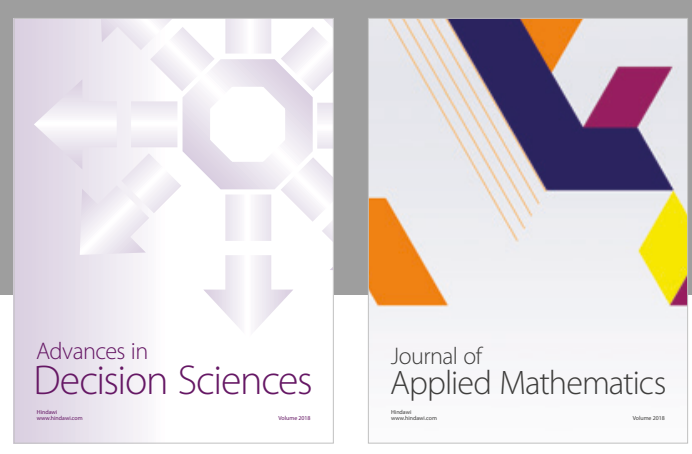

Journal of

Applied Mathematics
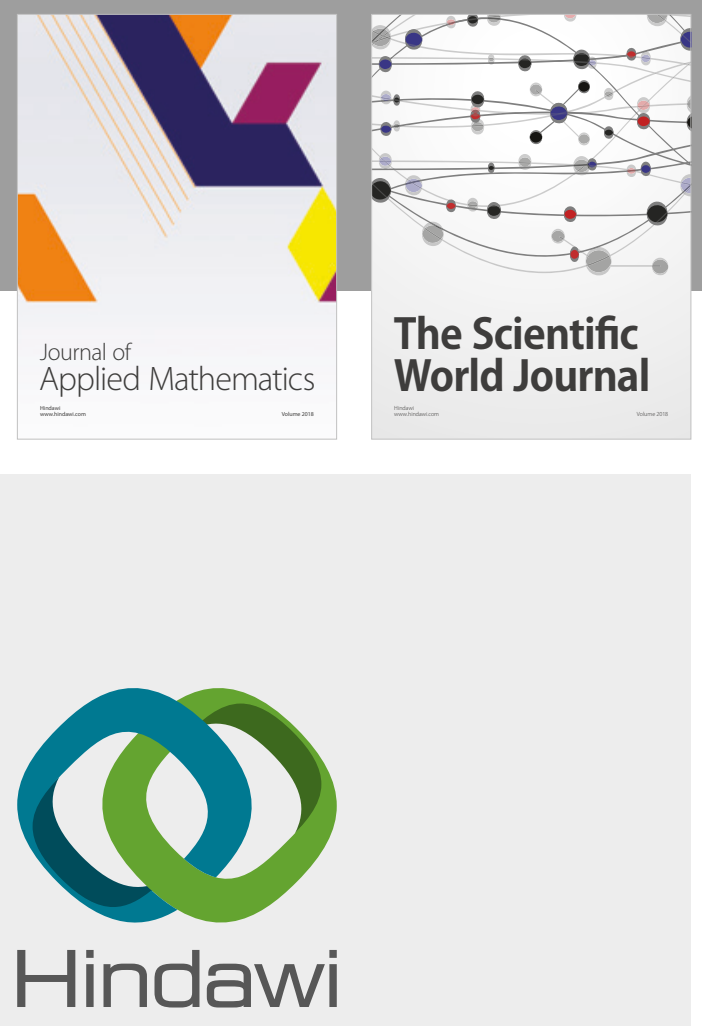

Submit your manuscripts at

www.hindawi.com

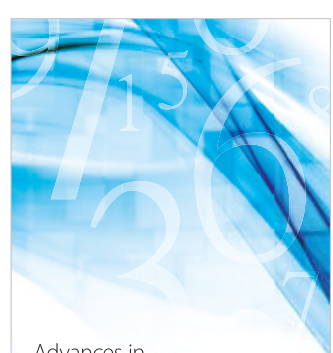

Advances in
Numerical Analysis
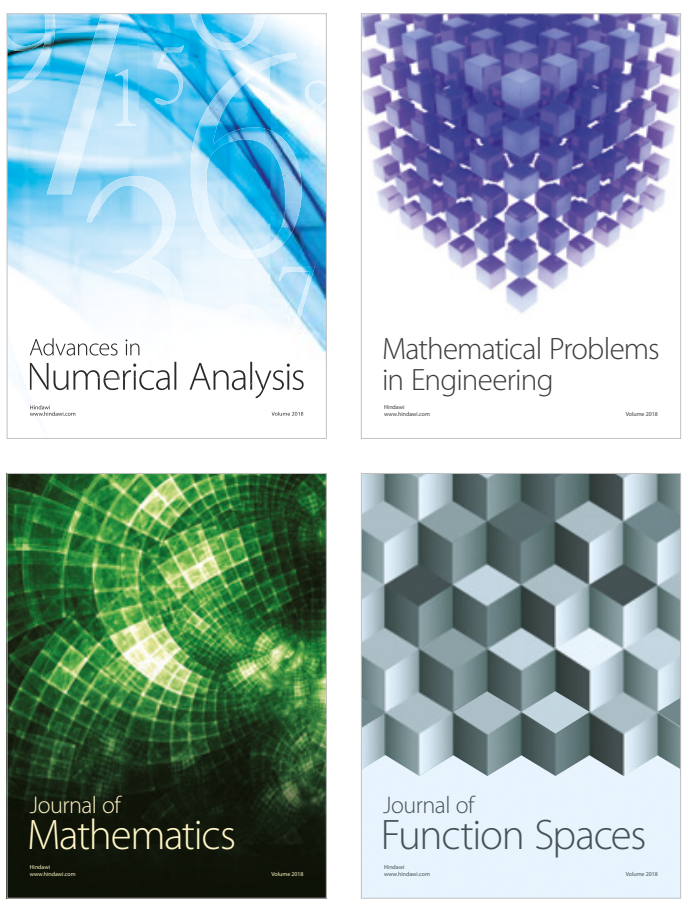

Mathematical Problems in Engineering

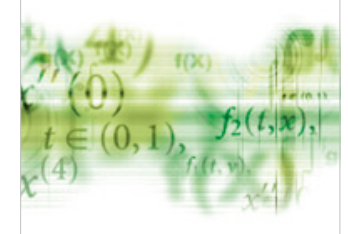

International Journal of

Differential Equations

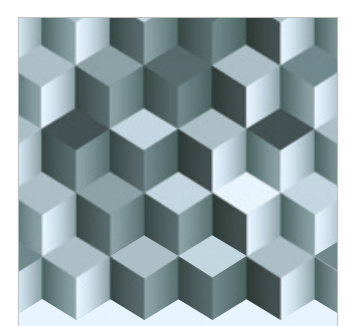

Journal of

Function Spaces

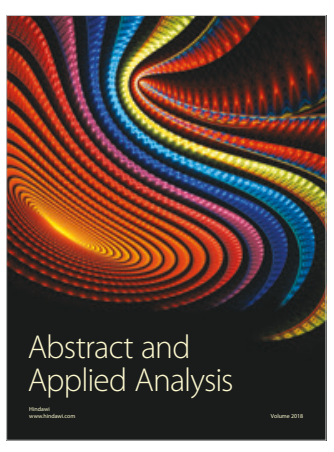

The Scientific

World Journal

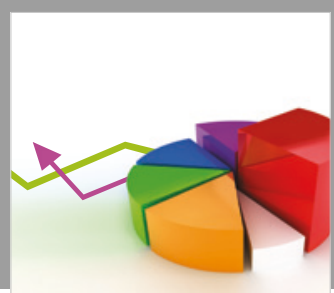

Journal of

Probability and Statistics
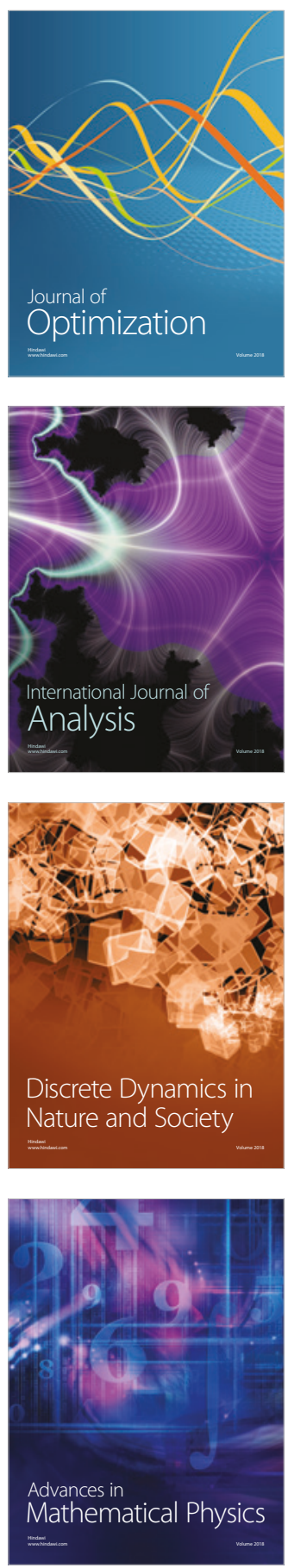